\title{
Limited correlation between tumor markers and minimal residual disease detected by seven neuroblastoma-associated mRNAs in high-risk neuroblastoma patients
}

\author{
SUGURU UEMURA ${ }^{1}$, KYAW SAN LIN ${ }^{1}$, KHIN KYAE MON THWIN ${ }^{1}$, NAOKO NAKATANI ${ }^{1}$, \\ TOSHIAKI ISHIDA ${ }^{2}$, NOBUYUKI YAMAMOTO ${ }^{1}$, AKIHIRO TAMURA ${ }^{2}$, ATSURO SAITO ${ }^{2}$, \\ TAKESHI MORI ${ }^{2}$, DAIICHIRO HASEGAWA ${ }^{2}$, YOSHIYUKI KOSAKA ${ }^{2}$, NANAKO NINO ${ }^{1}$, \\ CHINA NAGANO $^{1}$, SATORU TAKAFUJI ${ }^{1}$, KAZUMOTO IIJIMA $^{1}$ and NORIYUKI NISHIMURA ${ }^{3}$ \\ ${ }^{1}$ Department of Pediatrics, Kobe University Graduate School of Medicine, Kobe, Hyogo 650-0017; \\ ${ }^{2}$ Department of Hematology and Oncology, Kobe Children's Hospital, Kobe, Hyogo 650-0047; \\ ${ }^{3}$ Department of Public Health, Kobe University Graduate School of Health Science, Kobe, Hyogo 654-0142, Japan
}

Received August 3, 2020; Accepted December 18, 2020

DOI: $10.3892 / \mathrm{mco} .2021 .2299$

\begin{abstract}
Vanillylmandelic acid (VMA), homovanillic acid (HVA), neuron-specific enolase (NSE) and lactate dehydrogenase (LDH) are classical tumor markers and are used as standard clinical evaluations for patients with neuroblastoma (NB). Minimal residual disease (MRD) can be monitored by quantifying several sets of NB-associated mRNAs in the bone marrow (BM) and peripheral blood (PB) of patients with NB. Although MRD in BM and PB has been revealed to be a strong prognostic factor that is independent of standard clinical evaluations, its interrelation with tumor markers remains uncharacterized. The present study determined the levels of tumor markers (VMA, HVA, NSE and LDH) and MRD (BM-MRD and PB-MRD) in 133 pairs of concurrently collected BM, PB and urine samples from 19 patients with high-risk NB. The patients were evaluated during the entire course of treatment, which included 10 diagnoses, 32 treatments, 36 post-treatment, 9 relapses and 46 post-relapse sample pairs. The level of BM-MRD and PB-MRD was determined by quantifying $7 \mathrm{NB}-\mathrm{mRNAs}$ (collapsin response mediator protein 1, dopamine beta-hydroxylase, dopa decarboxylase, growth-associated protein 43, ISL LIM homeobox 1, pairedlike homeobox $2 \mathrm{~b}$ and tyrosine hydroxylase) using droplet digital PCR. In overall sample pairs, tumor markers (VMA, HVA, NSE and LDH) demonstrated weak but significant correlations $(\mathrm{P}<0.011)$ with $\mathrm{BM}-\mathrm{MRD}$ and $\mathrm{PB}-\mathrm{MRD}$.
\end{abstract}

Correspondence to: Dr Noriyuki Nishimura, Department of Public Health, Kobe University Graduate School of Health Science, 7-10-2 Tomogaoka, Suma-ku, Kobe, Hyogo 654-0142, Japan E-mail: nnishi@med.kobe-u.ac.jp

Key words: neuroblastoma, tumor markers, vanillylmandelic acid, homovanillic acid, neuron-specific enolase, lactate dehydrogenase, minimal residual disease, neuroblastoma-associated mRNAs
In subgroups according to each patient evaluation, the degree of correlation between tumor markers and MRD became stronger in patients with adrenal gland tumors, BM metastasis at diagnosis and relapse/regrowth compared with overall sample pairs. In contrast, tumor markers demonstrated variable correlations with MRD in subgroups according to each sample evaluation (BM infiltration at sampling, collection time point and disease status). The results suggested that tumor markers may demonstrate limited correlation with MRD in patients with high-risk NB.

\section{Introduction}

Neuroblastoma (NB) is the most common extracranial solid tumor in children and accounts for approximately $15 \%$ of pediatric cancer-associated deaths. It is characterized by extreme heterogeneity, ranging from spontaneous regression to malignant progression $(1,2)$. Although low- and intermediate-risk NB patients are successfully treated with $>90 \%$ long-term event-free survival rates, more than half of high-risk NB patients have experienced tumor relapse/regrowth and progression with a long-term survival rate of $40-50 \%(3,4)$. Patients with high-risk disease account for approximately half of newly diagnosed cases and are treated by the standard regimen consisting of induction chemotherapy, high-dose chemotherapy with autologous stem cell rescue, surgery, radiotherapy, and immunotherapy $(5,6)$. Despite these intensive multimodal therapies, as many as $20 \%$ of high-risk patients have residual disease that is refractory or progressive during induction chemotherapy $(7,8)$. The rest of the high-risk patients usually achieve remission, but the larger part of those patients has minimal residual disease (MRD) that causes relapse/regrowth and progression after completion of the standard regimen (9). Patients with relapse/regrowth are rarely cured, with $<10 \%$ long-term survival (10).

Vanillylmandelic acid (VMA) and homovanillic acid (HVA) levels in urine and neuron-specific enolase (NSE) and lactate dehydrogenase (LDH) levels in serum are classic 
tumor markers of NB (11). To date, NB has been diagnosed by elevated catecholamine metabolites (VMA and HVA), histopathology, and extensive imaging $(12,13)$. The International Neuroblastoma Staging System (INSS) also considered conventional serum markers (NSE and LDH) as an optional aid due to their limited diagnostic sensitivity and specificity (13). Although tumor markers (VMA, HVA, NSE, and LDH) were well characterized at initial diagnosis and during response assessment, their prognostic value in predicting tumor relapse/regrowth and progression was far from optimal (14). Indeed, the International Neuroblastoma Risk Group (INRG) classification system and the International Neuroblastoma Response Criteria (INRC) considered no tumor markers as diagnostically sensitive/specific enough to be incorporated as risk factors or response criteria, respectively $(15,16)$. Despite these limitations, tumor markers are widely used as standard clinical evaluations for NB patients in current clinical practice.

To achieve optimal outcomes for high-risk NB patients, an accurate evaluation of MRD is essential to monitor the disease burden and treatment response. An increasing number of MRD assays using different methods and samples has been reported. For instance, immunophenotyping with flow cytometry (FCM) and detection of DNA methylation were applied to detect $\mathrm{MRD}$ in NB patients. NB patients with $\mathrm{NB} 84^{+} / \mathrm{CD}^{2} 6^{+} / \mathrm{CD} 45$ or $\mathrm{GD} 2^{+} / \mathrm{CD} 1^{+} / \mathrm{CD} 6^{+} / \mathrm{CD} 45^{-}$cells in $\mathrm{BM}$ samples had significantly poor outcomes compared to NB patients without $\mathrm{NB} 4^{+} / \mathrm{CD} 6^{+} / \mathrm{CD} 45^{-}$or $\mathrm{GD} 2^{+} / \mathrm{CD} 81^{+} / \mathrm{CD} 56^{+} / \mathrm{CD} 45^{-}$cells in BM samples $(17,18)$. DNA methylation of the tumor suppressor RASSF1A was reported to be a highly specific DNA marker for MRD detection and is better suited for MRD quantification in BM samples (19). However, MRD in NB patients was most commonly identified by detecting neuroblastoma-associated mRNAs (NB-mRNAs) by quantitative PCR (qPCR) due to the absence of recurrent oncogenic-fusion genes in NB cells $(20,21)$. Whereas single NB-mRNA (TH or PHOX2B mRNA) was initially evaluated in BM and PB samples (22-24), multiple NB-mRNAs were later used to achieve more sensitive MRD detection. Several sets of NB-mRNAs have been reported to have significant prognostic value for NB patients (25-28). However, these sets used considerably different NB-mRNAs and resulted in a significant but limited prognostic power with low accuracy $(0.5<$ area under curve $($ AUC) $<0.7)(28,29)$. We initially validated 14 NB-mRNAs (included in all multiple marker sets of NB-mRNAs) and selected 11 NB-mRNAs based on their expression in spheres of NB cells by qPCR, which enabled the early detection of relapse/regrowth in two high-risk NB cases $(30,31)$. We then developed a new MRD assay that quantified 7NB-mRNAs (CRMP1, DBH, DDC, GAP43, ISL1, PHOX2B, and TH mRNAs) using droplet digital PCR (ddPCR). This ddPCR-based MRD assay outperformed the qPCR-based MRD assay, and the level of 7NB-mRNAs in $\mathrm{BM}$ predicted tumor relapse/regrowth with moderate accuracy $(0.7<$ AUC <0.9) (32).

Although MRD in BM and PB (BM-MRD and PB-MRD) has been shown to be a strong prognostic factor independent of standard clinical evaluations (28), its interrelation with tumor markers remains uncharacterized. In the present study, we determined the levels of tumor markers (VMA, HVA, NSE and $\mathrm{LDH}$ ) and MRD (BM-MRD and PB-MRD) in 133 pairs of concurrently collected BM, PB, and urine samples from 19 high-risk NB patients during the entire course of treatment, and examined their interrelation in overall sample pairs and subgroups of sample pairs.

\section{Patients and methods}

NB patients and samples. Nineteen high-risk NB patients defined by the Children's Oncology Group (COG) Neuroblastoma Risk Stratification System $(2,33)$ or the INRG Classification System (15) were included in this study. All patients were diagnosed and treated at Kobe Children's Hospital or Kobe University Hospital between June 2011 and January 2018 based on the JN-H-11 (UMIN000005045) or JN-H-15 (UMIN000016848) protocol of the Japanese Children's Cancer Group (JCCG) Neuroblastoma Committee (JNBSG). All BM, PB, and urine samples were collected concurrently ( $<7$ days apart) as frequently as possible during the entire course of treatment. This study was approved by the Ethics Committee of Kobe University Graduate School of Medicine and Kobe Children's Hospital and was conducted in accordance with the guidelines for Clinical Research of Kobe University Graduate School of Medicine. Written informed consent was obtained from all patients.

Disease evaluation. Disease evaluation was conducted as described previously (32). Briefly, evaluation was conducted at every collection time point in accordance with the INRC based on the available medical records $(13,34)$. Responses were assigned to 'remission' corresponding to complete response (CR) or very good partial response (VGPR), 'stable' corresponding to partial response (PR), mixed response (MR), or no response (NR), or 'progression' corresponding to progressive disease (PD) for all BM, PB, and urine sample pairs.

Tumor marker. Tumor marker data (VMA and HVA in urine, NSE, and LDH in serum) were extracted from the medical records. All patients were subjected to a food/drink restriction (bananas, citrus fruits, coffee, vanilla-containing confectionery, and salicylic acid preparations are on the list) for urine sample collection. A spot urine sample in the early morning was used to measure VMA and HVA. Normal reference ranges of VMA, HVA, NSE, and LDH were 1.2-4.9 $\mu \mathrm{g} / \mathrm{mg}$ creatinine, 1.6-5.5 $\mu \mathrm{g} / \mathrm{mg}$ creatinine, $0-16.3 \mathrm{ng} / \mathrm{ml}$, and $124-270 \mathrm{IU} / \mathrm{l}$ for most children, respectively.

$M R D$ in $B M$ and $P B$. BM and $\mathrm{PB}$ sample preparation and 7NB-mRNA ddPCR assays were performed as described previously (32). Briefly, all BM and PB samples were separated using Mono-Poly resolving medium (DS Pharma Biomedical), and nucleated cells were collected. Total RNA was extracted with a TRIzol Plus RNA purification kit (Life Technologies; Thermo Fisher Scientific, Inc.). cDNA was synthesized from 1 or $0.5 \mu \mathrm{g}$ total RNA using a Quantitect reverse transcription kit (Qiagen $\mathrm{GmbH}$ ) and stored at $-80^{\circ} \mathrm{C}$ until use. The 7NB-mRNA ddPCR assay measured the expression of 7NB-mRNAs (CRMP1, DBH, DDC, GAP43, ISL1, PHOX2B, and TH mRNA) and a reference gene mRNA (HPRT1 mRNA) with optimized probe and primer sets, cDNA, and standard 
thermal cycling conditions using a QX200 ddPCR system (Bio-Rad Laboratories, Inc.) according to the digital Minimum Information for Publication of Quantitative Digital PCR Experiments guidelines $(35,36)$. The level of 7NB-mRNAs (combined signature) was defined as the weighted sum of 7 relative copy numbers (level of each NB-mRNA), in which the reciprocal of the 90th percentile in non-NB control samples was used for the weighting for each NB-mRNA. For BM samples, the level of 7NB-mRNAs was calculated as the mean of the right and left samples.

Statistical analysis. Correlation between the level of 7NB-mRNAs in BM and PB samples and the levels of VMA, HVA, NSE, and LDH in urine and PB samples was assessed using Spearman's rank correlation test. Reported P-values are two-sided, and $\mathrm{P}<0.05$ was considered statistically significant. Correlation coefficient $r$ values were interpreted as follows: 0.00-0.09 as 'negligible', 0.10-0.39 as 'weak', 0.40-0.69 as 'moderate', $0.70-0.89$ as 'strong', and 0.90-1.00 as 'very strong' according to a previous report (37). EZR (version 1.35, www.jichi.ac.jp/saitama-sct/SaitamaHP.files/statmedEN. html; Saitama Medical Center, Jichi Medical University, Saitama, Japan), which is a modified version of $\mathrm{R}$ commander designed to add statistical functions that is frequently used in biostatistics (38) was used for statistical analyses.

\section{Results}

Characteristics of $B M, P B$, and urine sample pairs. A total of $133 \mathrm{BM}, \mathrm{PB}$, and urine sample pairs collected concurrently ( $<7$ days apart) from 19 high-risk NB patients were enrolled in the present study. As summarized in Table I, all sample pairs were subdivided into subgroups according to each sample or patient evaluation. For each sample evaluation, all BM samples were pathologically analyzed by histology/immunohistochemistry (IHC) of biopsies or cytology/immunocytology (IC) of aspirates according to the INRC recommendations (34). Consequently, BM infiltration at sampling was present in 21 samples and absent in 112 samples. Sample pairs were drawn as frequently as possible during the entire course of high-risk NB treatment, and the collection time points were defined as 'diagnosis' at initial diagnosis, 'treatment' including induction chemotherapy, high-dose chemotherapy with autologous peripheral blood stem cell transplantation (PBSCT), surgery, and radiation, 'post-treatment' including 13-cis-retinoic acid (13-cis-RA) treatment and follow-up before relapse, 'relapse' at relapse diagnosis, and 'post-relapse' including all treatments and follow-up after relapse. Consequently, samples were subdivided into 10 diagnosis, 32 treatment, 36 post-treatment, 9 relapse, and 46 post-relapse samples. Disease status at sampling was retrospectively evaluated as remission, stable and and progression. Consequently, samples were subdivided into 21 remission, 87 stable, and 25 progression samples (Table I). For each patient evaluation, all sample pairs were subdivided according to the following prognostic factors: Age at diagnosis, primary tumor site, BM metastasis at diagnosis, DNA ploidy, MYCN status, relapse/regrowth, and recurrent tumor site. Consequently, 80\% (107/133) were derived from $\geq 18$ month old patients, $80 \%$ (106/133) from patients with an adrenal grand tumor, 92\% (122/133) from
Table I. Patient and sample characteristics.

Variable

Patients, n (\%) Samples, n (\%)

BM infiltration at sampling ${ }^{\mathrm{a}}$

Present

10

$21(16)$

Absent

16

$112(84)$

Collection time point of

sample ${ }^{a}$

$\begin{array}{lrr}\text { Diagnosis } & 10 & 10(7) \\ \text { Treatment } & 9 & 32(24) \\ \text { Post-treatment } & 10 & 36(27) \\ \text { Relapse } & 8 & 9(7) \\ \text { Post-relapse } & 9 & 46(35)\end{array}$

Disease status of sample ${ }^{\mathrm{a}}$

Remission

$21(16)$

Stable

6

$87(65)$

Progression

13

25 (19)

Sex $^{\mathrm{b}}$

Male

13 (68)

87

Female

$6(32)$

46

Age at diagnosis ${ }^{\mathrm{b}}$

$<18$ months

3 (16)

26

$\geq 18$ months

$16(84)$

107

Primary tumor site ${ }^{b}$

Adrenal gland

Non-adrenal gland

$14(74)$

106

5 (26)

$\mathrm{BM}$ metastasis at diagnosis ${ }^{\mathrm{b}}$

Present

$16(84)$

122

Absent

3 (16)

11

Histopathology

Favorable

$\begin{array}{ll}0 & (0)\end{array}$

0

Unfavorable

$19(100)$

133

DNA ploidy ${ }^{\mathrm{b}}$

Diploid

14 (74)

93

Hyperdiploid

3 (16)

2 (10)

33

Unknown

MYCN status ${ }^{b}$

Amplified

7 (37)

10

Non-amplified

12 (63)

45

Relapse/regrowth ${ }^{\mathrm{b}}$

Present

11 (58)

8 (42)

90

Absent

4 (36)

35

CNS

$7(64)$

Non-CNS

${ }^{a}$ The percentage was not calculated for patient data. ${ }^{\mathrm{b}}$ The percentage was not calculated for sample data and a single patient was classified into multiple categories. BM, bone marrow; MYCN, MYCN proto-oncogene, bHLH transcription factor; CNS, central nervous system.

patients with BM metastasis at diagnosis, 70\% (93/126) from patients with diploid tumors, 34\% (45/133) from patients with MYCN-amplified tumors, 68\% (90/133) from patients with 
Tumor markers and BM-MRD
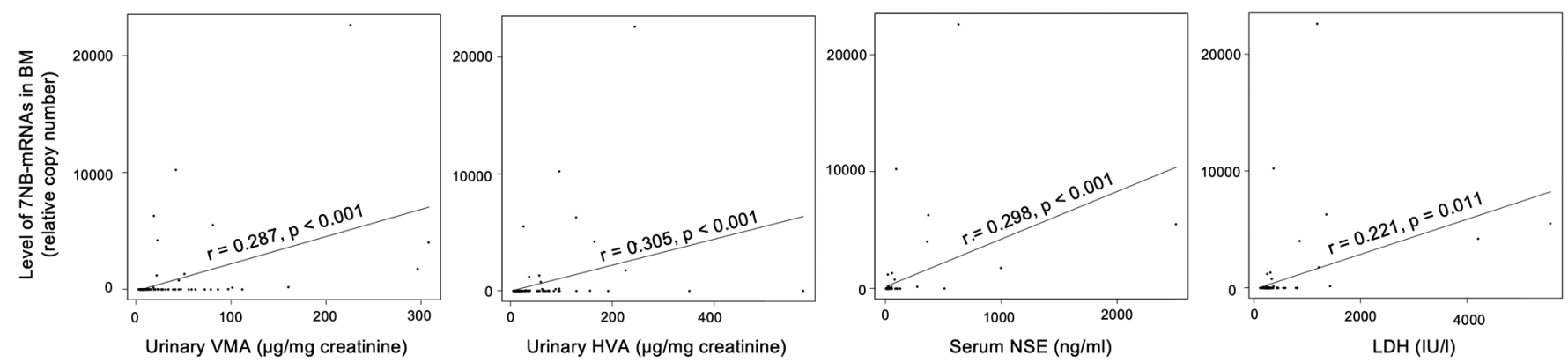

Tumor markers and PB-MRD
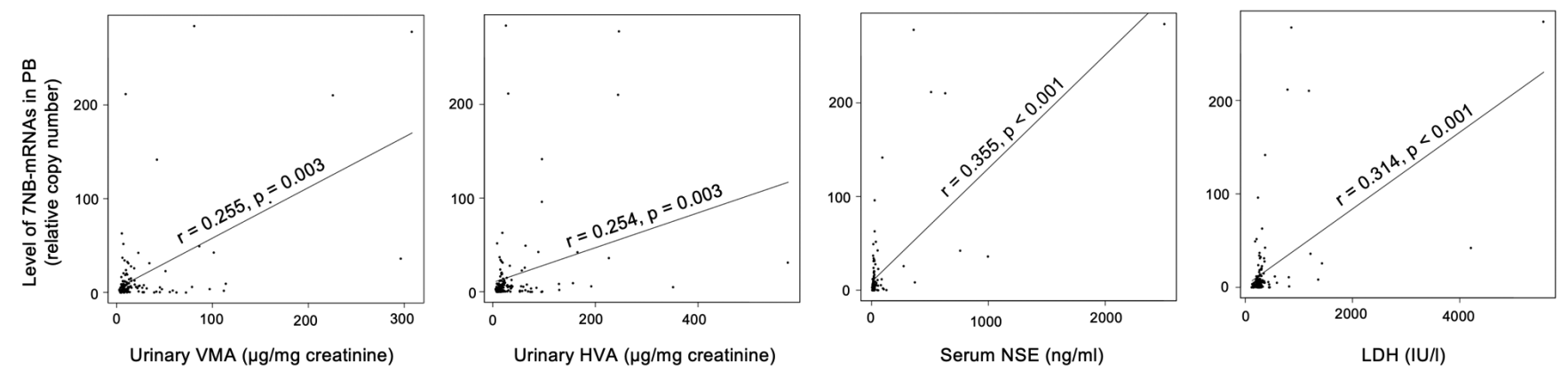

Figure 1. Correlations between tumor markers and MRD in overall sample pairs. The levels of 7 neuroblastoma-mRNA (relative copy number) were determined by droplet digital polymerase chain reaction and its correlations with the levels of VMA $(\mu \mathrm{g} / \mathrm{mg}$ creatinine), $\mathrm{HVA}(\mu \mathrm{g} / \mathrm{mg} \mathrm{creatinine}), \mathrm{NSE}(\mathrm{ng} / \mathrm{ml})$ and LDH (IU/l) were assessed by Spearman's rank correlation coefficient in 133 pairs of concurrently collected BM, PB and urine samples. MRD, minimal residual disease; VMA, vanillylmandelic acid; HVA, homovanillic acid; NSE, neuron-specific enolase; LDH, lactate dehydrogenase; BM, bone marrow; $\mathrm{PB}$, peripheral blood.

relapse/regrowth, and 39\% (35/90) from CNS-relapse/regrowth patients (Table I).

Correlations between tumor markers and MRD in overall sample pairs. We first analyzed the correlations of tumor markers (VMA, HVA, NSE, and LDH) with MRD (BM-MRD and PB-MRD) in 133 overall sample pairs. VMA (3.3-308.3, median $10.0 \mu \mathrm{g} / \mathrm{mg}$ creatinine), HVA (6.2-575.8, median $19.5 \mu \mathrm{g} / \mathrm{mg}$ creatinine), NSE (8.2-2,510.0, median $21.6 \mathrm{ng} / \mathrm{ml})$, and LDH (128-5,567, median $274 \mathrm{IU} / \mathrm{l})$ showed weak but significant correlations with both BM-MRD (0.1-22, 630.8, median 3.2 copies) and PB-MRD (0.1-284.5, median 5.9 copies). Very similar degrees of correlation ( $\mathrm{r}=0.221-0.355$, $\mathrm{P}<0.03)$ were detected among different combinations of tumor markers (VMA, HVA, NSE, and LDH) and MRD (BM-MRD and PB-MRD) (Fig. 1).

Correlations between tumor markers and MRD in subgroups according to each patient's evaluation. To characterize the observed correlations between tumor markers (VMA, HVA, NSE, and LDH) and MRD (BM-MRD and PB-MRD) in overall sample pairs, we then analyzed them in subgroups according to each patient's evaluation: Age at diagnosis, primary tumor site, BM metastasis at diagnosis, DNA ploidy, MYCN status, relapse/regrowth, and recurrent tumor site (Table I). Among these evaluations, primary tumor site, $\mathrm{BM}$ metastasis at diagnosis, and relapse/regrowth showed a constant impact on the degrees of correlations between tumor markers (VMA, HVA, NSE, and LDH) and MRD (BM-MRD and PB-MRD). In subgroups of patients with adrenal gland tumors, the correlations of VMA, HVA, NSE, and LDH with BM-MRD became stronger than the overall sample pairs. The same was true for PB-MRD. The correlations of tumor markers (VMA, HVA, NSE, and LDH) with MRD (BM-MRD and PB-MRD) also became stronger than the overall sample pairs in subgroups of patients with BM metastasis at diagnosis and with relapse/regrowth. In contrast, tumor markers did not show statistically significant correlations with MRD in subgroups of patients without adrenal gland tumors, BM metastasis at diagnosis, and in patients without relapse/regrowth (Fig. 2).

Correlations between tumor markers and MRD in subgroups according to each sample evaluation. Next, we analyzed the correlations between tumor markers (VMA, HVA, NSE, and LDH) and MRD (BM-MRD and PB-MRD) in subgroups according to each sample evaluation: BM infiltration at sampling, collection time point, and disease status (Table I). In subgroups according to BM infiltration at sampling, the correlation became moderate or strong $(r=0.49-0.821, \mathrm{P}<0.026)$ in the positive subgroup and non-significant in the negative subgroup, except for a weak correlation $(r=0.228, \mathrm{P}=0.016)$ between NSE and PB-MRD in the negative subgroup. In subgroups according to the collection time point, the correlations between tumor markers (VMA, HVA, NSE, and LDH) and MRD (BM-MRD and PB-MRD) varied considerably in the diagnosis, treatment, post-treatment, relapse, and post-relapse 
Primary tumor site
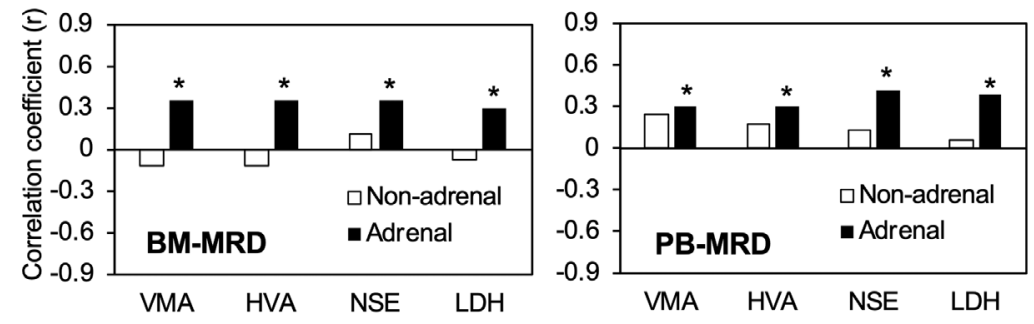

BM metastasis at diagnosis
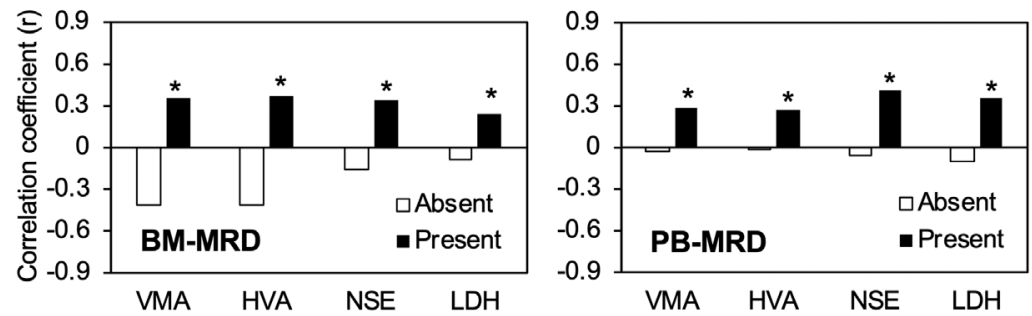

Relapse/regrowth
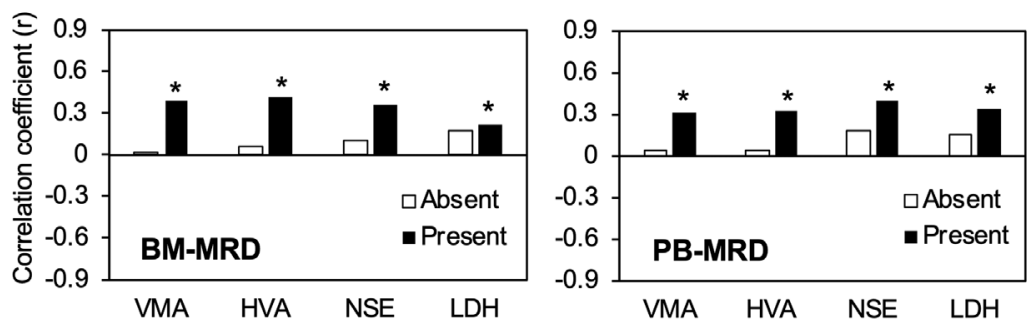

Figure 2. Correlations between tumor markers and MRD in subgroups of sample pairs according to each patient evaluation. Levels of 7 neuroblastoma-mRNA (relative copy number) were determined by droplet digital polymerase chain reaction and its correlations with the levels of VMA ( $\mu \mathrm{g} / \mathrm{mg}$ creatinine), HVA ( $\mu \mathrm{g} / \mathrm{mg}$ creatinine), NSE (ng/ml) and LDH (IU/l) were assessed by Spearman's rank correlation coefficient in subgroups of concurrently collected BM, PB and urine sample pairs: Primary tumor site (non-adrenal, 27 pairs; adrenal, 106 pairs), BM metastasis at diagnosis (absent, 11 pairs; present, 122 pairs) and relapse/regrowth (absent, 43 pairs; present, 90 pairs). ${ }^{*} \mathrm{P}<0.05$ (significant correlation). MRD, minimal residual disease; VMA, vanillylmandelic acid; HVA, homovanillic acid; NSE, neuron-specific enolase; LDH, lactate dehydrogenase; BM, bone marrow; PB, peripheral blood.

subgroups. In subgroups according to disease status, the correlations between tumor markers (VMA, HVA, NSE, and LDH) and MRD (BM-MRD and PB-MRD) also varied substantially in the remission, stable, and progression subgroups (Fig. 3).

\section{Discussion}

In the present study, we determined the levels of tumor markers (VMA, HVA, NSE, and LDH) and MRD (BM-MRD and $\mathrm{PB}-\mathrm{MRD}$ ) in 133 pairs of concurrently collected BM, PB, and urine samples from 19 high-risk NB patients during the entire course of treatment. Representative data obtained from a typical relapsed NB patient were presented in Fig. S1. To avoid multiple testing, we designed the present study as a descriptive study that did not set an endpoint for the correlation between tumor markers and MRD, and showed that tumor markers had limited correlations with MRD in high-risk NB patients. The correlation was weak but significant in overall sample pairs and was more constantly affected by patient factors (primary tumor site, BM metastasis at diagnosis, and relapse/regrowth) than sample factors (BM infiltration at sampling, disease status, and collection time point).

VMA, HVA, NSE, and LDH are currently used as standard clinical evaluations of NB despite their limitations in diagnostic sensitivity/specificity for risk factors/response criteria $(15,16,39)$. MRD (BM-MRD and PB-MRD) can be monitored by quantifying 7NB-mRNAs (CRMP1, DBH, DDC, GAP43, ISL1, PHOX2B, and TH mRNA) using ddPCR (32). Among the four tumor markers and seven NB-mRNAs, two tumor markers (VMA and HVA) are catecholamine metabolites and three NB-mRNAs (DBH, DDC, and TH mRNA) are the transcripts of catecholamine biosynthetic enzymes. Elevated levels of tumor markers (VMA, HVA, NSE, and LDH) and MRD (BM-MRD and PB-MRD) are associated with the existence and/or increase of tumor burden $(9,11)$. Although MRD has been shown to be a strong prognostic factor independent of standard clinical evaluations (28), the interrelation of MRD (BM-MRD and PB-MRD) with tumor markers (VMA, HVA, NSE, and LDH) has never been characterized during the course of treatment in high-risk NB patients. The present results revealed that tumor markers showed a weak but significant correlation with MRD in overall sample pairs, including all stages of high-risk NB treatment (Fig. 1). While the level of PB-MRD was substantially lower than that of BM-MRD $(28,32)$, the levels of BM-MRD and PB-MRD were equally correlated with tumor markers in urine (VMA and HVA) and PB (NSE and LDH) (BM-MRD: $r=0.221-0.305$, $\mathrm{P}<0.01$, PB-MRD: $\mathrm{r}=0.254-0.355, \mathrm{P}<0.03)$. 
BM infiltration at sampling
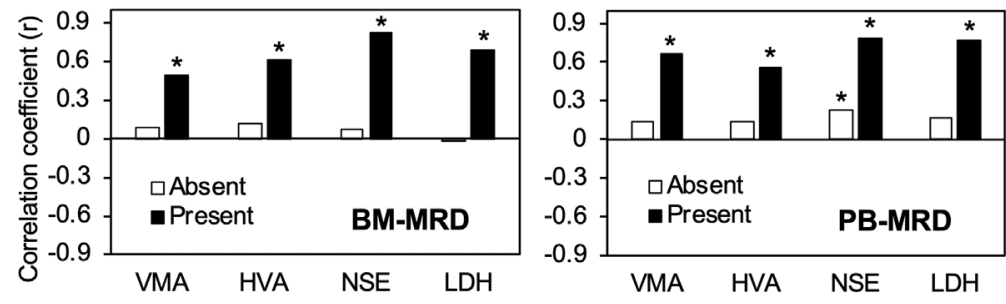

Collection time point
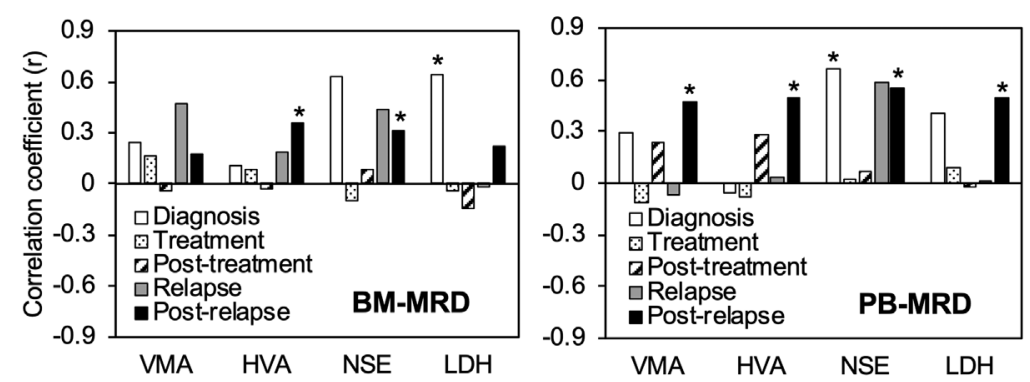

Disease status
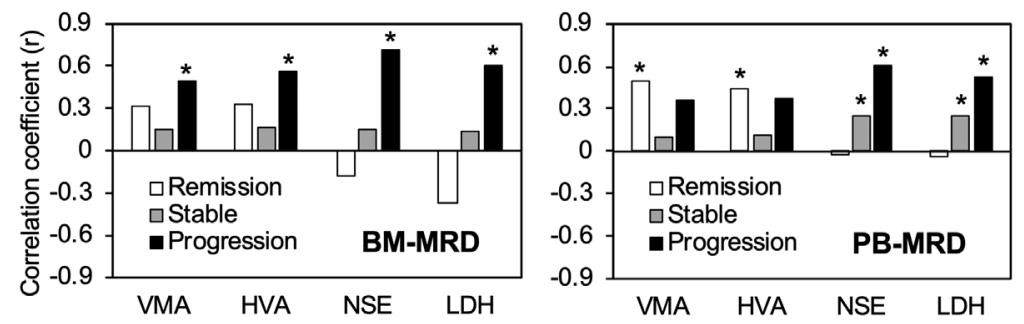

Figure 3. Correlations between tumor markers and MRD in subgroups of sample pairs according to each sample evaluation. Correlations between tumor markers and MRD in subgroups of sample pairs according to each patient evaluation. Levels of 7 neuroblastoma-mRNA (relative copy number) were determined by droplet digital polymerase chain reaction and its correlations with the levels of VMA ( $\mu \mathrm{g} / \mathrm{mg} \mathrm{creatinine}), \mathrm{HVA}(\mu \mathrm{g} / \mathrm{mg} \mathrm{creatinine}), \mathrm{NSE}$ (ng/ml) and LDH (IU/1) were assessed by Spearman's rank correlation coefficient in subgroups of concurrently collected BM, PB and urine sample pairs: BM infiltration at sampling (absent, 112 pairs; present, 21 pairs), collection time point (diagnosis, 10 pairs; treatment, 32 pairs; post-treatment, 36 pairs; relapse, 9 pairs; post-relapse, 46 pairs) and disease status (remission, 21 pairs; stable, 87 pairs; progression, 25 pairs). ${ }^{*} \mathrm{P}<0.05$ (significant correlation). MRD, minimal residual disease; VMA, vanillylmandelic acid; HVA, homovanillic acid; NSE, neuron-specific enolase; LDH, lactate dehydrogenase; BM, bone marrow; $\mathrm{PB}$, peripheral blood.

The most striking result of the present study was a constant impact of patient factors on the degree of interrelation between tumor markers (VMA, HVA, NSE, and LDH) and MRD (BM-MRD and PB-MRD) (Fig. 2). As each patient had 1-19 sample pairs (12 patients had >6 sample pairs) to be analyzed, subgroups of sample pairs were generated according to the following patient factors: Primary tumor site, BM metastasis at diagnosis, relapse/regrowth, age at diagnosis, DNA ploidy, MYCN status, and the recurrent tumor site (Figs. 2 and S2). Among these patient factors, three (primary tumor site, BM metastasis at diagnosis, and relapse/regrowth) had a constant impact on the degree of correlation between tumor markers and MRD (Figs. 2 and S2). In contrast, a variable impact of sample factors (BM infiltration at sampling, disease status, and collection time point) was found in the present study (Fig. 3). Since the existence and/or increase of tumor burden has been shown to be associated with elevated levels of both tumor markers and MRD, it is naturally assumed that their association would be influenced by their levels. However, disease status and collection time point did not have a constant impact on their correlation, although BM infiltration at sampling had a considerable impact. Taken together, patient factors intrinsic to patients/tumors rather than sample factors representing the status at sampling might be involved in the interrelation between tumor markers and MRD in high-risk NB patients.

However, there were limitations to this study. The small number of patients and samples is a limitation of this study. Another limitation is that a part of patient factors (age at diagnosis, DNA ploidy, MYCN status, and recurrent tumor site) show variable impacts on the correlations between tumor markers (VMA, HVA, NSE, and LDH) and MRD (BM-MRD and PB-MRD). The reason for the different impacts of patient factors is not clear at present; thus, further studies are required.

In summary, there are limited correlations between tumor markers (VMA, HVA, NSE, and LDH) and MRD (BM-MRD and PB-MRD) in high-risk NB patients. Although the interrelations between tumor markers and MRD vary substantially among subgroups of sample pairs, 
the interrelations are more constantly affected by patient factors rather than sample factors. Prospective studies with scheduled/pre-determined sample collection time points, clinical studies with uniform treatment strategies, and studies with a larger numbers of NB patients and samples, ensuring minimal bias, are required to validate the present results

\section{Acknowledgements}

The authors would like to thank Dr Tomoko Fujikawa at the Pediatric Department of Kobe University Hospital and Dr Sayaka Nakamura, Dr Machiko Miyamoto, Dr Jun Noguchi, Dr Ryunosuke Tojyo, Dr Shotaro Inoue and Dr Akihiro Nishimura at the Hematology and Oncology Department at Kobe Children's Hospital for collecting BM, PB and urine samples.

\section{Funding}

The present study was supported in part by Grants-in-Aid for Scientific Research (KAKENHI) from the Japan Society for the Promotion of Science (grant nos. 17K10110, 19K17361 and 19K17331) and the Institutional Research Fund of Sysmex Corporation to Kobe University.

\section{Availability of data and materials}

All data generated or analyzed during this study are included in this published article.

\section{Authors' contributions}

SU, KSL, KKMT and NNis. drafted the initial manuscript. SU, KSL, KKMT, DH, YK, KI and NNis conceptualized and designed the study. SU, KSL, KKMT, NNak, TI, NY, AT, AS, TM, NNin, CN, and ST collected the samples, acquired the data and carried out analyses. SU, KSL, KKMT and NNis performed statistical analysis and interpreted the data. SU, KSL, DH, YK, KI and NNis critically revised the article for important intellectual content. All authors have read and approved the final manuscript.

\section{Ethics approval and consent to participate}

The present study was approved by the Ethics Committee of Kobe University Graduate School of Medicine (approval no. 180278) and Kobe Children's Hospital (approval no. 30-80). Informed consent to participate in the present study was obtained from all patients.

\section{Patient consent for publication}

Not applicable.

\section{Competing interests}

NNis received institutional research funding from Sysmex Corporation to Kobe University. The remaining authors declare that they have no competing interests.

\section{References}

1. Brodeur GM: Neuroblastoma: Biological insights into a clinical enigma. Nat Rev Cancer 3: 203-216, 2003.

2. Maris JM,Hogarty MD, Bagatell R and Cohn SL: Neuroblastoma. Lancet 369: 2106-2120, 2007.

3. Pinto NR, Applebaum MA, Volchenboum SL, Matthay KK, London WB, Ambros PF, Nakagawara A, Berthold F, Schleiermacher G, Park JR, et al: Advances in risk classification and treatment strategies for neuroblastoma. J Clin Oncol 33: 3008-3017, 2015.

4. Morgenstern DA, Potschger U, Moreno L, Papadakis V, Owens C, Ash S, Pasqualini C, Luksch R, Garaventa A, Canete A, et al: Risk stratification of high-risk metastatic neuroblastoma: A report from the HR-NBL-1/SIOPEN study. Pediatr Blood Cancer 65: e27363, 2018.

5. Smith V and Foster J: High-risk neuroblastoma treatment review. Children (Basel) 5: 114, 2018

6. MacFarland S and Bagatell R: Advances in neuroblastoma therapy. Curr Opin Pediatr 31: 14-20, 2019.

7. Maris JM: Recent advances in neuroblastoma. N Engl J Med 362: 2202-2211, 2010

8. Tolbert VP and Matthay KK: Neuroblastoma: Clinical and biological approach to risk stratification and treatment. Cell Tissue Res 372: 195-209, 2018.

9. Uemura S, Ishida T, Thwin KKM, Yamamoto N, Tamura A Kishimoto K, Hasegawa D, Kosaka Y, Nino N, Lin KS, et al: Dynamics of minimal residual disease in neuroblastoma patients. Front Oncol 9: 455, 2019.

10. London WB, Castel V, Monclair T, Ambros PF, Pearson AD, Cohn SL, Berthold F, Nakagawara A, Ladenstein RL, Iehara T and Matthay KK: Clinical and biologic features predictive of survival after relapse of neuroblastoma: A report from the International Neuroblastoma Risk Group project. J Clin Oncol 29: 3286-3292, 2011.

11. Riley RD: A systematic review of molecular and biological tumor markers in neuroblastoma. Clin Cancer Res 10: 4-12, 2004.

12. Brodeur GM, Seeger RC, Barrett A, Berthold F, Castleberry RP, D'Angio G,De Bernardi B, Evans AE, Favrot M, Freeman AI, et al: International criteria for diagnosis, staging, and response to treatment in patients with neuroblastoma. J Clin Oncol 6: 1874-1881, 1988.

13. Brodeur GM, Pritchard J, Berthold F, Carlsen NL, Castel V, Castelberry RP, De Bernardi B, Evans AE, Favrot M, Hedborg F, et al: Revisions of the international criteria for neuroblastoma diagnosis, staging, and response to treatment. J Clin Oncol 11: 1466-1477, 1993.

14. Simon T,Hero B, Hunneman DH and Berthold F: Tumour markers are poor predictors for relapse or progression in neuroblastoma. Eur J Cancer 39: 1899-1903, 2003.

15. Cohn SL, Pearson ADJ, London WB, Monclair T, Ambros PF, Brodeur GM, Faldum A, Hero B, Iehara T, Machin D, et al: The international neuroblastoma risk group (INRG) classification system: An INRG task force report. J Clin Oncol 27: 289-297, 2009.

16. Park JR, Bagatell R, Cohn SL, Pearson AD, Villablanca JG, BertholdF, Burchill S, Boubaker A, McHugh K, Nuchtern JG, et al: Revisions to the international neuroblastoma response criteria: A consensus statement from the national cancer institute clinical trials planning meeting. J Clin Oncol 35: 2580-2587, 2017.

17. Bozzi F, Gambirasio F, Luksch R, Collini P, Brando B and Fossati-Bellani F: Detecting CD $56^{+} / \mathrm{NB} 84^{+} / \mathrm{CD} 45$ immunophenotype in the bone marrow of patients with metastatic neuroblastoma using flow cytometry. Anticancer Res 26: 3281-3287, 2006.

18. Popov A, Druy A, Shorikov E, Verzhbitskaya T, Solodovnikov A, Saveliev L, Tytgat GAM, Tsaur G and Fechina L: Prognostic value of initial bone marrow disease detection by multiparameter flow cytometry in children with neuroblastoma. J Cancer Res Clin Oncol 145: 535-542, 2019.

19. Stutterheim J, Ichou FA, den Ouden E, Versteeg R, Caron HN, Tytgat GA and van der Schoot CE: Methylated RASSF1a is the first specific DNA marker for minimal residual disease testing in neuroblastoma. Clin Cancer Res 18: 808-814, 2012.

20. Beiske K, Ambros PF, Burchill SA, Cheung IY and Swerts K: Detecting minimal residual disease in neuroblastoma patients-the present state of the art. Cancer Lett 228: 229-240, 2005.

21. Brownhill SC and Burchill SA: PCR-based amplification of circulating RNAs as prognostic and predictive biomarkers-Focus on neuroblastoma. Pract Lab Med 7: 41-44, 2017. 
22. Burchill SA, Bradbury FM, Smith B, Lewis IJ and Selby P: Neuroblastoma cell detection by reverse transcriptase-polymerase chain reaction (RT-PCR) for tyrosine hydroxylase mRNA. Int J Cancer 57: 671-675, 1994.

23. Viprey VF, Corrias MV, Kågedal B, Oltra S, Swerts K, Vicha A, Ladenstein R and Burchill SA: Standardisation of operating procedures for the detection of minimal disease by QRT-PCR in children with neuroblastoma: Quality assurance on behalf of SIOPEN-R-NET. Eur J Cancer 43: 341-350, 2007.

24. Stutterheim J, Gerritsen A, Zappeij-Kannegieter L, Kleijn I, Dee R, Hooft L, van Noesel MM, Bierings M, Berthold F, Versteeg R, et al: PHOX2B is a novel and specific marker for minimal residual disease testing in neuroblastoma. J Clin Oncol 26: 5443-5449, 2008.

25. Stutterheim J, Zappeij-Kannegieter L, Versteeg R, Caron HN van der Schoot CE and Tytgat GAM: The prognostic value of fast molecular response of marrow disease in patients aged over 1 year with stage 4 neuroblastoma. Eur J Cancer 47: 1193-1202, 2011.

26. Viprey VF, Gregory WM, Corrias MV, Tchirkov A, Swerts K, Vicha A, Dallorso S, Brock P, Luksch R, Valteau-Couanet D, et al: Neuroblastoma mRNAs predict outcome in children with stage 4 neuroblastoma: A European HR-NBL1/SIOPEN study. J Clin Oncol 32: 1074-1083, 2014

27. Cheung NK, Ostrovnaya I, Kuk D and Cheung IY: Bone marrow minimal residual disease was an early response marker and a consistent independent predictor of survival after anti-GD2 immunotherapy. J Clin Oncol 33: 755-763, 2015.

28. Marachelian A, Villablanca JG, Liu CW, Liu B, Goodarzian F, Lai HA, Shimada H, Tran HC, Parra JA, Gallego R, et al: Expression of five neuroblastoma genes in bone marrow or blood of patients with relapsed/refractory neuroblastoma provides a new biomarker for disease and prognosis. Clin Cancer Res 23: 5374-5383, 2017.

29. Akobeng AK: Understanding diagnostic tests 3: Receiver operating characteristic curves. Acta Paediatr 96: 644-647, 2007.

30. Hartomo TB, Kozaki A, Hasegawa D, Van Huyen Pham T, Yamamoto N, Saitoh A, Ishida T, Kawasaki K, Kosaka Y, Ohashi H, et al: Minimal residual disease monitoring in neuroblastoma patients based on the expression of a set of real-time RT-PCR markers in tumor-initiating cells. Oncol Rep 29: 1629-1636, 2013.

31. Hirase S, Saitoh A, Hartomo TB, Kozaki A, Yanai T, Hasegawa D, Kawasaki K, Kosaka Y, Matsuo M, Yamamoto N, et al: Early detection of tumor relapse/regrowth by consecutive minimal residual disease monitoring in high-risk neuroblastoma patients. Oncol Lett 12: 1119-1123, 2016.
32. Thwin KKM, Ishida $T$, Uemura $S$, Yamamoto $N$, Lin $K S$, Tamura A, Kozaki A, Saito A, Kishimoto K, Mori T, et al: Level of seven neuroblastoma-associated mRNAs detected by droplet digital PCR is associated with tumor relapse/regrowth of high-risk neuroblastoma patients. J Mol Diagn 22: 236-246, 2020.

33. Weinstein JL, Katzenstein HM and Cohn SL: Advances in the diagnosis and treatment of neuroblastoma. Oncologist 8: 278-292, 2003.

34. Burchill SA, Beiske K, Shimada H, Ambros PF, Seeger R, Tytgat GA, Brock PR, Haber M, Park JR and Berthold F: Recommendations for the standardization of bone marrow disease assessment and reporting in children with neuroblastoma on behalf of the International Neuroblastoma Response Criteria Bone Marrow Working Group. Cancer 123: 1095-1105, 2017.

35. Bustin SA, Benes V, Garson JA, Hellemans J, Huggett J, Kubista M, Mueller R, Nolan T, Pfaffl MW, Shipley GL, et al: The MIQE guidelines: Minimum information for publication of quantitative real-time PCR experiments. Clin Chem 55: 611-622, 2009.

36. Huggett JF, Foy CA, Benes V, Emslie K, Garson JA, Haynes R, Hellemans J, Kubista M, Mueller RD, Nolan T, et al: The digital MIQE guidelines: Minimum information for publication of quantitative digital PCR experiments. Clin Chem 59: 892-902, 2013.

37. Schober P, Boer C and Schwarte LA: Correlation coefficients. Anesth Analg 126: 1763-1768, 2018.

38. Kanda Y: Investigation of the freely available easy-to-use software 'EZR' for medical statistics. Bone Marrow Transplant 48 452-458, 2013.

39. Moroz V, Machin D, Hero B, Ladenstein R, Berthold F, Kao P, Obeng Y, Pearson ADJ, Cohn SL and London WB: The prognostic strength of serum LDH and serum ferritin in children with neuroblastoma: A report from the International Neuroblastoma Risk Group (INRG) project. Pediatr Blood Cancer 67: e28359, 2020. 